\title{
Terahertz Computed Tomographic Reconstruction and its Wavelet-based Segmentation by Fusion
}

\author{
X.X. Yin, B.W.-H. Ng, B. Ferguson, S.P. Mickan, D. Abbott \\ School of Electrical \& Electronic Engineering, The University of Adelaide, SA 5005, Australia \\ xxyin@eleceng.adelaide.edu.au
}

\begin{abstract}
In this paper, terahertz (T-ray) computed tomographic (CT) imaging [1] and segmentation techniques are investigated. The traditional filtered back projection is applied for the reconstruction of terahertz coherent tomography. A set of linear image fusion and novel wavelet scale correlation segmentation techniques is adopted to achieve material discrimination within a three dimensional (3D) object. The methods are applied to a T-ray CT image dataset taken from a plastic vial containing a plastic tube. This setup simulates the imaging of a simple nested organic structure, which provides an indication of the potential for using T-ray CT imaging to achieve T-ray pulsed signal classification of heterogeneous layers. The wavelet based fusion scheme enjoys the additional benefit that it does not require the calculation of a single threshold and there is only a segmenter parameter to adjust.
\end{abstract}

\section{INTRODUCTION}

'T-rays' is a collective term to describe the part of the electromagnetic spectrum from $0.1 \mathrm{THz}$ to $10 \mathrm{THz}$. The application of T-rays, especially in biomedicine and quality control, is attractive owing to two intrinsic properties: a non-ionising nature and penetration through dry, non-polar and non-metallic materials. Rapid improvements in T-ray detectors and sources make it possible to image objects through optically opaque layers. Currently, most THz pulsed imaging (TPI) is based on the extraction of material parameters, which are calculated based on (i) time-of-flight measurements in reflection and transmission geometry or (ii) the variation in amplitude or phase of the transmitted waveform at certain frequencies. $\mathrm{THz}$ tomographic reconstruction is another interesting application of T-ray imaging. It exploits methods from geophysical, radar and optical diffraction techniques [1]. They are capable of mapping the $2 \mathrm{D}$ and $3 \mathrm{D}$ distribution of scattering objects, but with a constraint to image the shape profile of the target object. At present, three dimensional (3D) T-ray CT imaging, described in [1], has been developed based on coherent T-ray detection techniques, which allows the imaging of the internal structure and extract the frequency dependent properties in three dimensions. Along with a dimension in time, this makes it possible 4D datasets of the target sample.

Current two-dimensional and three- dimensional T-ray CT imaging is affected by different types of noise, which can restrict its usefulness [2]. The result of the noise is that the acquisition conditions determine the received T-ray intensities and similar substance shows various responses in different portions of the same image. These drawbacks have prevented successful development of accurate and reliable edge-detection and segmentation algorithms for T-ray CT images. A reliable and automatic segmentation detection is of great importance in many applications of T-ray image processing, especially for the T-ray pulsed signal classification of 3D objective CT imaging with heterogeneous layers. (Handley et. al. 2002) employed clustering techniques for segmentation with wavelet preprocessing and a synthetic and a real $\mathrm{THz}$ slice are used for the segmentation evaluation [3]. In comparison, our current job utilises wavelet techniques to achieve segmentation and the analysis of internal structure of the target object is used to evaluate the segmentation quality.

The current wavelet scale correlation based segmentationby-fusion algorithms afford another approach in 3D T-ray tomographic imaging to probe the interior regions of objects. In conjunction with various edge detection techniques, our algorithm provides quantitative information for measurement and classification applications. Identification of a linear shift in the computed centroids of each height is used for segmentation evaluation, due to the strait internal structure of the plastic tube. One of the main advantages of the algorithm is that, in its simplest form, it requires only a single segmentation parameter. In addition, a wavelet based segment-by-fusion detection method for T-ray images is effective with respect to noise.

The motivation for wavelet based segmentation is to achieve the discrimination of T-ray pulsed signals within a 3D heterogeneous layers. It is observed that there exists difficulty to divide the transmitted pulse via time slice for three classes: the vial, the tube and the background, due to the lack of obvious variety of peak and valley after the penetration of $\mathrm{T}$ rays through three target objects. This is because, at different rotation angles and for different tested objects, there are different refractive, scattering and absorption coefficients, which result in a great decrease in one of the peak values of the response and a relatively large increase in the other. A simple consideration for the slice of different target classes is the application of segmentation techniques to T-ray CT functional imaging, which affords an effective approach to define the regions for different classes, i.e. classification of T-ray CT images for functional imaging.

This paper contains the two main sections: methodology and experimental results. The methodology section describes the hardware and algorithms employed in this work. Section 2.1 describes the chirped probe pulse $\mathrm{THz}$ imaging system, which forms the basis of the 3D T-ray CT system. Reconstruction 


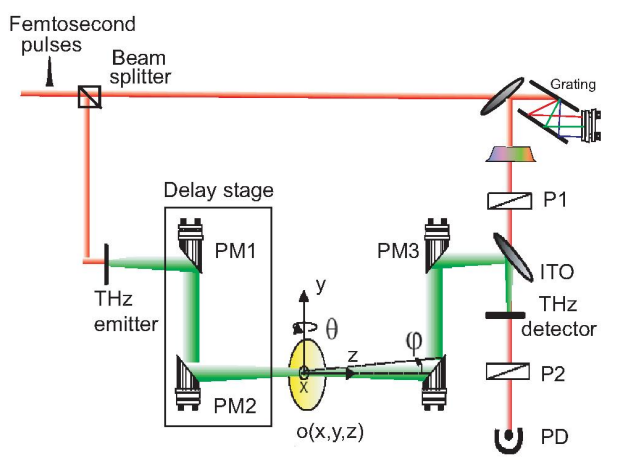

Fig. 1. A chirped probe pulse $\mathrm{THz}$ imaging system for the realisation of a 3D T-ray CT imaging. After [1].

algorithms are discussed in Section 2.2, and Section 2.3 describes a novel method to achieve reconstructed segmentation, called wavelet scale correlation based segmentati-on-byfusion. Section 3 consists of the experimental segmentation results and the evaluation of segmentation quality. Section 4 concludes this paper.

\section{The Methodology}

\section{A. A Brief Introduction to T-ray Functional Imaging}

A 3D T-ray CT system is based on the standard THz-TDS scanned imaging system illustrated in Fig. 1. The target is mounted on a motion stage that allows it to be translated by $\mathrm{T}$ rays in $x$ and $z$ axes. Meanwhile, the object can be rotated and linearly moved along the $y$ axis. As seen in Fig. 1, Terahertz pulsed imaging (TPI) is achieved by repeating pulsed Terahertz measurements in a 2D raster scan. An ultrafast pulsed laser beam is split into separate probe and pump beams. The path length of the pump beam is modulated by a delay stage, then transmitted through a chopper and enters one of the optical rectification crystals, which acts as a T-ray emitter. The Trays produced are recollimated and focused onto a sample by a pair of optical lenses. The T-rays emerging from the sample are adjusted again by another pair of optical lenses before being combined with the probe beam. As a result, the T-ray response and the probe beams propagate through the $\mathrm{THz}$ detector crystal co-linearly. For the current chirped pulsed imaging system, the optical probe beam is chirped by a grating pair and allows an electro-optic crystal to sample the $\mathrm{THz}$ temporal profile simultaneously. The detector crystal produces optical outputs that are proportional to the T-ray response, and the signals are measured with the use of a photodetector. Based on the different peak times of the measured pulses, sets of 3-D volume data of an object can be obtained for different object angles and the change of the position along the $x$ and $z$ axes, once the measurement process is completed.

In this experiment, a four-dimensional (4D) dataset is acquired with polar coordinates $(\theta, l, y, t)$, where $\theta$ is the projection angle, $y$ is the vertical axis (rotation axis), $l$ is the perpendicular distance from projection path to rotation axis, and $t$ is the sampled time. The corresponding rectangular coordinate system in the frequency domain, transformed from

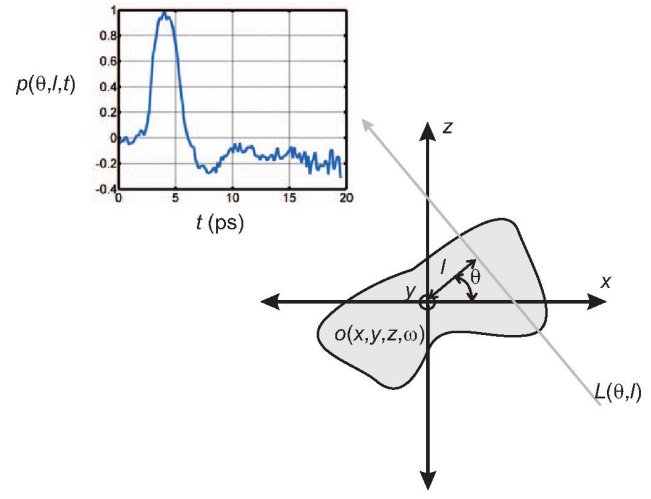

Fig. 2. Two sets of coordinate systems for the reconstructed T-ray CT. A typical $\mathrm{THz}$ pulse spectrum is shown as a function of time $t$ in the inset. After [1].

the polar coordinate system, labeled by $(x, y, z, \omega)$ is employed for the reconstruction of the object's optical properties, where $\omega$ indicates the frequency, and the $x, y$ and $z$ axes represent standard Cartesian coordinates (the $y$ axis is perpendicular to the page). The processing required to effect this transformation is described in section 2.2 below. The resolution for each slice image reaches $1 \mathrm{~mm}$, and the T-ray CT images have a resolution of $89 \times 89$ pixels. For further details on the experiments, please refer to [1].

\section{B. An Overview of $C T$ and T-ray $C T$}

Computed tomography produces sectional images of an object, which has important application to capture internal organs or structures in its actual 3D position. A filtered back projection algorithm, used for the calculation of the inverse Radon transform [4], [1], is employed to reconstruct a target object. The quantities obtained are the object's frequency dependent optical properties.

Normally, a filtered back projection algorithm can be realised geometrically by propagating a sinogram image back into image space, with a ramp filter to diminish noise and discretisation artefacts (see Fig. 3(b)). A sinogram is also called Radon transform data, denoted below by $\Re$ and is simply a collection of the projections at all the projection angles. A 1D projection operation at each projection angle is a linear integral of the image intensity along projection path $L$, which satisfies the following function:

$$
p(l, \theta)=\int o(x, z) \mathrm{d} L=\Re(o)
$$

where all points on projection path $L$ satisfy the equation: $x \cos \theta+z \sin \theta=l$. Fig. 3(a) shows the radon projection.

Compared to X-ray computed tomographic techniques, a Tray $\mathrm{CT}$ experiment is capable of measuring the transmitted $\mathrm{THz}$ pulse as a function of time $t$, for a given projection angle and projection offset $p_{d}(t, \theta, l)$. The Fourier transform of this time domain pulse yields $P_{d}(\omega, \theta, l)$. The reference pulse $p_{i}(t)$ and the corresponding Fourier response $P_{i}(\omega)$ can be measured by removing the target object from background. If 


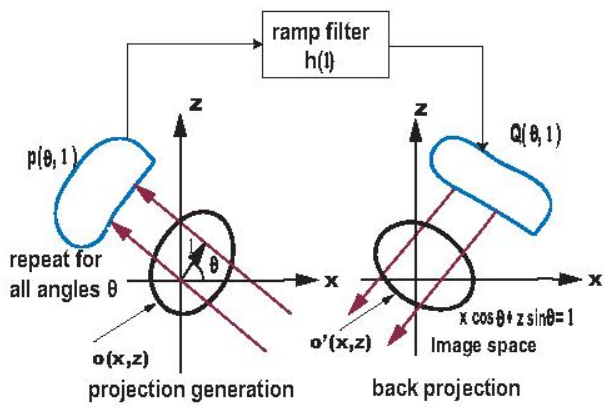

Fig. 3. Illustration of the geometric paths regarding a general scheme of computed tomography. (a) The geometric path of $1 \mathrm{D}$ projection operation. (b) The geometric paths regarding filter back projection operation.

the target is rotated and propagated by T-ray beams, $P_{d}(\omega, \theta, l)$ may be evaluated by adding sufficient projection angles to allow the filtered back projection algorithm to be applied at each specific frequency $\omega$. This is based on the approximation that the detected $\mathrm{THz}$ signal is viewed as a linear integral of the incident $\mathrm{THz}$ pulse,

$$
P_{d}(\omega, \theta, l)=P_{i}(\omega) \exp \left[\int_{L(\theta, l)} \frac{-i \omega \hat{n}(r)}{c} \mathrm{~d} r\right]
$$

where $P_{d}$ and $P_{i}$ are the Fourier transforms of the detected and incident THz signals, respectively; $c$ is the speed of light, $L$ is the projection path, a straight line between the source and detector. The unknown complex refractive index of the sample is noted by $\hat{n}(\omega, r)=n_{\delta}(\omega, r)+i k(\omega, r)$, where $n_{\delta}(\omega, r)$ is the real refractive index deviation and $k(\omega, r)$ is the extinction coefficient, related to absorption coefficient $\alpha$ via $k(\omega, r)=$ $\alpha / 2 k_{i}$ ( $k_{i}$ is the incident extinction coefficient). Let us define that

$$
\begin{gathered}
P_{n} \doteq \arg \left[\frac{P_{d}(\theta, l)}{P_{i}(\theta, l)}\right] / k_{i}=\int_{L} n_{\delta}(r) d r=\Re\left\{n_{\delta}(r)\right\} \\
P_{\alpha} \doteq-2\left\|\frac{P_{d}(\theta, l)}{P_{i}(\theta, l)}\right\|=\int_{L} \alpha(r) d r=\Re\{\alpha(r)\} .
\end{gathered}
$$

where $\arg (x)$ denotes the phase or argument of complex valued $x,\|x\|$ denotes the magnitude of the complex scalar $x$, and $P_{n}$ and $P_{\alpha}$ are the projection data inputs to the filtered back projection algorithm as required to reconstruct $n_{\delta}$ and $\alpha$, respectively, at a specific THz frequency $\omega$. The sign $r$ denotes the position of the incident field (the sensor).

The filtered back projection algorithm for T-ray CT reconstruction is expressed as follows:

$$
s(x, y)=\int_{0}^{\pi}\left[\int_{-\infty}^{\infty} S(\theta, \xi)|\xi| \exp [i 2 \pi \xi l] \mathrm{d} \xi\right] \mathrm{d} \theta .
$$

where $S(\theta, \xi)$ is the spatial Fourier transform of the parallel projection data, defined as

$$
S(\theta, \xi)=\int_{-\infty}^{\infty} s(\theta, l) \exp [-i 2 \pi \xi l] \mathrm{d} \xi,
$$

here, $s(\theta, l)$ is the measured projection data, $\xi$ is the spatial frequency in the $l$ direction. One of the advantages of $\mathrm{T}$ ray CT over X-ray CT is that $s(\theta, l)$ may be one of several parameters derived from the $\mathrm{THz}$ pulses. For instance, either Eq. 3 or Eq. 4 can substitute for $s(\theta, l)$. The choice can be made based on the desired application. In order to obtain a 3D image reconstruction, each reconstructed horizontal slice and surface is rendered for the identification of objects in a 3D volume space. T-ray CT has the potential to identify targets based on their frequency responses. The full frequencydependent reconstruction algorithm described above can be applied to a vial sample to demonstrate frequency-dependent $3 \mathrm{D}$ reconstruction.

Fig. 6(a) shows the two output $\mathrm{THz}$ pulsed responses measured in a time scan of optical delay line; the dash line is viewed as a reference pulse. Fig. 6(b) shows the log spectrum of the time-domain waveforms in (a). The black solid line indicates the de-convolved Fourier spectrum that is achieved via dividing the Fourier coefficients of detected signal (object) by the those of the incident signal (free-space). The de-convolved Fourier spectrum can be viewed as a one dimensional projection of the $\mathrm{THz}$ signal. The oscillations which follow the two main pulses, and which appear as dips on the three T-ray spectra, are a result of water vapor in the beam path. From the spectra, it is evident that the useable bandwidth of the signals is limited to $0.5 \mathrm{THz}$, because most of the source power is at frequencies below $0.5 \mathrm{THz}$. Therefore the reconstructions at higher frequencies suffer from additional artifacts caused by noise.

The object photograph of a plastic vial containing a different plastic tube, simulates a simple nested structure - as shown in Fig. 4(a). The target is imaged with a $1 \mathrm{~mm}$ step size in the $x$ and $y$ dimensions, and at projections separated by $10^{\circ}$. First the reconstruction is performed using the timing of the peak of the $\mathrm{THz}$ pulse in the time domain to yield a reconstruction of the bulk absorption coefficients. For the current experiment, the real part of the expression $\log \left[P_{d}(\omega) / P_{i}(\omega)\right]$ is used to reconstruct T-ray $\mathrm{CT}$ at each sampled frequency. The central slice is reconstructed at each of the ten lowest frequencies, from 0.0213 to $0.213 \mathrm{THz}$, (illustrated in Fig. 5) chosen for the strong signals at those frequencies. The 3D rendered image is illustrated in Fig. 4(b), which is the combination of the reconstructed slices at a number of target heights. The resulting isosurface is constructed using the pixels where the reconstructed absorption coefficients are evaluated. The reconstructed image dimensions are quite accurate, with the vial and cylinder diameters being within $15 \%$ of the actual dimensions measured with calipers. However, the vial thickness is much thicker than expected because of the coarse reconstruction grid size of $1.5 \mathrm{~mm}$. The grid size may be improved using more projection angles.

\section{Wavelet Based Segmentation by Fusion}

As described above, T-ray CT extracts the frequency dependent 3D characteristics of a target sample. This makes possible rich, four dimensional datasets that describe the sample. To 


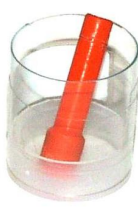

(a)

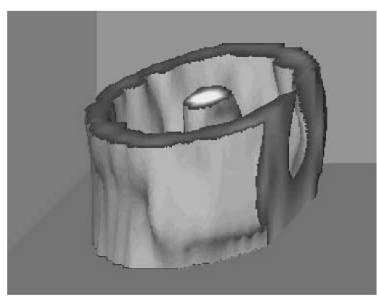

(b)
Fig. 4. A vial containing a plastic tube is used as a simple nested structure to test the T-ray CT system and to demonstrate the resulting reconstruction and classification of the T-ray CT imaging. (a) The optical image of the vial and the tube. (b) The 3D reconstruction of the vial and tube based on the T-ray CT slices.

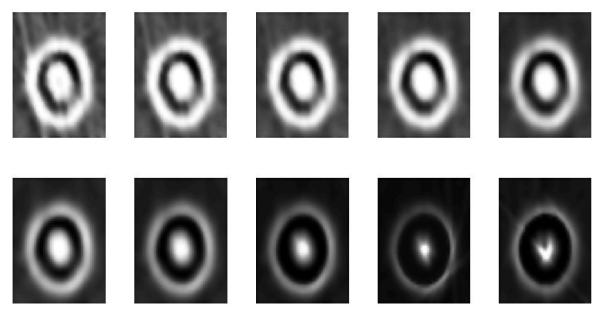

Fig. 5. Illustration of the reconstructed T-ray CT slices at the first 10 frequencies, in increasing order from top left at the object height of $7 \mathrm{~mm}$.

achieve the final T-ray CT image, an inverse Radon transform (IRT) is computed on the Fourier coefficients of the measured signals. In order to obtain the material discrimination within a three dimensional (3D) object, a set of linear image fusion and novel two dimensional (2D) wavelet scale correlation segmentation are adopted. The methods are applied to a T-ray CT image dataset of a plastic vial containing a plastic tube, with an aim to simulate the imaging of a simple nested organic structure. The setup is imaged at various heights, ranging from $5 \mathrm{~mm}$ to $9 \mathrm{~mm}$ (from the bottom), in $1 \mathrm{~mm}$ increments. Image fusion algorithms are conducted on the low frequency reconstructed T-ray CT images at different target heights for smooth version of CT images. In turn, 2D discrete wavelet transform (DWT) is taken on the fused image. In order to reconstruct the same size of the approximate sub-images as the fused image, inverse discrete wavelet transform (IDWT) is performed after the appropriate subband is isolated. The target segments are localised by computing the correlation between the first two approximate scales sub-images.

\section{1) Image Fusion of T-ray CT Images for a 3D Target:}

A fused image can be created via emerging two or more images, acquired from a single source to obtain the sharp display of various materials and structure [5], [6]. Our segmentation techniques is based on a plane fusion approach to match and fuse multiple desirable slice images at various frequencies into a single slice image. The current image fusion is conducted at pixel level and the recovered images at the different sampled

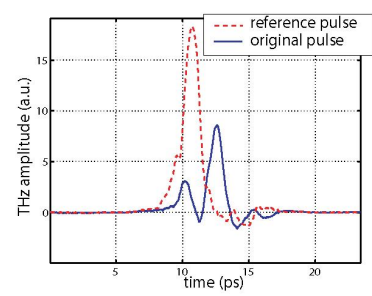

(a)

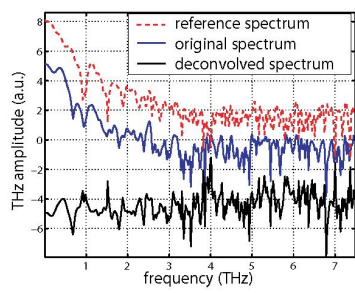

(b)
Fig. 6. Illustration of T-ray signals and spectra. (a) Detected T-ray signal and reference; (b) their spectra in $\log$ scale with offsets of 2 a.u. and 4 a.u. corresponding to the original and de-convolved spectra, respectively, for clarity.

frequencies at the same measurement height presents complementary information about the measured visual physical parameters at the region investigated.

The proposed fusion scheme processes images via the following steps. A linear combination of weighted slice images is used to perform plane image fusion in the current experiment for enhanced contrast of features and reduced inconstancy. The weights are selected based on the various intensity for the different frequency of T-ray CT images. In this work, the weighting scheme is empirically chosen to be $1 / 15$ for the five lower frequencies and $2 / 15$ for the five higher frequencies to compensate for the attenuation in signal strength in the higher frequency components and to highlight the consistency in the boundaries. In this way the fusion takes place at each measurement height and the more dominant features at each sampled frequency is preserved (ten sampled frequencies are illustrated for this paper). Subsequently a new image is constructed by emerging the ten weighted images. The resultant fused image has clear target contours and shows strong contrast between the target regions and the background. Fig. 7(a) shows the resultant fused image with size of $89 \times 89$.

\section{2) Discrete Wavelet Transforms in Two Dimensions:}

The 2D DWT can be realised by digital filters and downsampling the T-ray image. Expansion by a 2D scaling function and three $2 \mathrm{D}$ wavelet functions are calculated by taking the 1D fast wavelet transform (FWT) of the rows of an image and the resulting columns [7]. This algorithm can be inverted via upsampling the signals, filtering and adding the output together to recover the low-frequency subband at the previous level of the multiresolution analysis. The process is repeated for the depth of the DWT to obtain the original image. In our work, however, the individual subbands at the lowest resolution are isolated (i.e. all other subbands at the same level are set to zero) before application of the inverse wavelet transform (IWT). This processing allows the wavelet scale correlation based segmentation to be performed (section 2.3.3).

The block diagram, see Fig. 8 shows the 2D wavelet transform procedure.

Fig. 9(a) and (b) are the reconstructed sub-images after tak- 


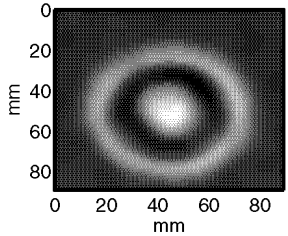

(a)

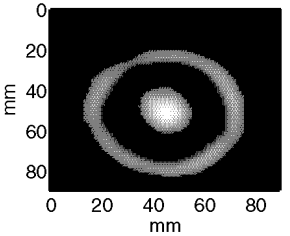

(b)
Fig. 7. Illustration of reconstructed central slice T-ray CT images. (a) Fused image via merging T-ray CTs at ten lowest frequencies. (b) Final extracted object segments from the background using wavelet based segmentation by fusion.

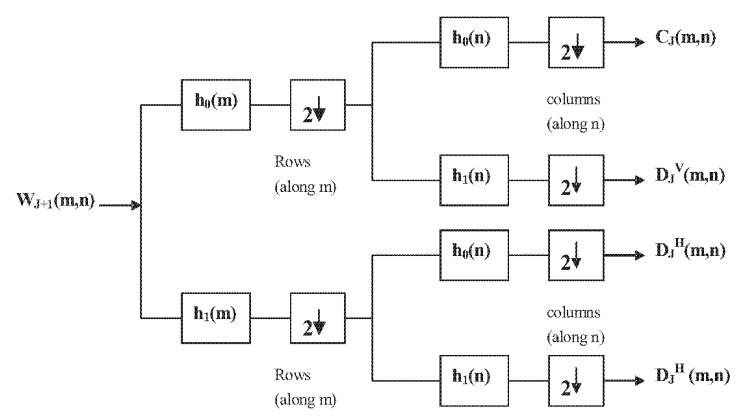

Fig. 8. Illustrate the 2D discrete wavelet transform procedure. After [8].

ing 2D inverse wavelet transform of approximate coefficients at the first and second wavelet decomposed scales. The T-ray fused image at a $7 \mathrm{~mm}$ target height is used. A Daubechies 4 wavelet as a mother wavelet is applied for this case. In each group of the two scales of reconstructed T-ray CT subimages, the first sub-image is the reconstructed approximate sub-image and the other three are detail reconstructed subimages. It can be seen that the approximate sub-images are the smooth version of original image.

\section{3) 2D Wavelet Scale Correlation Based Segmentation:}

The aim of segment detection is to achieve differentiated subdivision of constituent regions of an image. The method used in this paper is motivated by one dimensional wavelet scale correlation denoising. Firstly, the target cross-sections are assumed to be corrupted by additive white Gaussian noise, which is randomly distributed. The target objects are separated by their absorption coefficients, which are indicated by the intensity in the images. With the incremental wavelet scale, the noise is reduced and the target intensity (energy) is increased in an image. After extracting the low frequency 2D subband of fused T-ray CT images, an increased energy with an increase in wavelet scale is used as a cue to extract the target regions. This cue is consistent with the procedure used in 1D wavelet de-noising, where the noise usually appear as small coefficients in the wavelet subbands. By computing a correlation of two scales of wavelet subbands, the signal
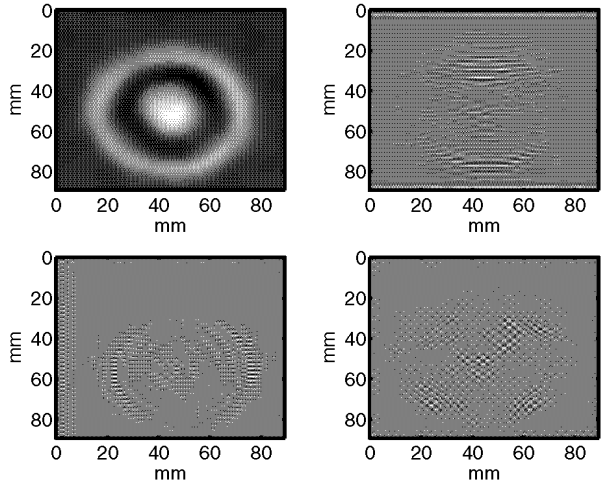

(a)
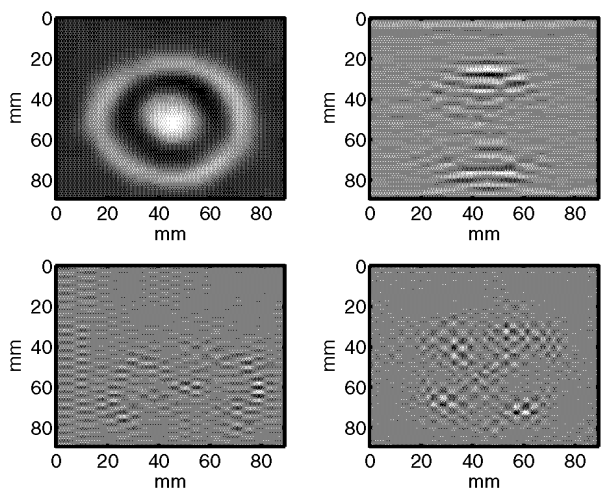

(b)

Fig. 9. Illustration of the sub-images via reconstructed approximation coefficients. (a) The reconstructed approximate sub-image after one-scale wavelet decomposition of the fused T-ray CT. (b) The reconstructed approximate subimage after two-scale wavelet decomposition of the fused T-ray CT.

components should survive in large coefficients while the noise would be diminished. The wavelet scale correlation based segmentation algorithm is summarized as:

1. Calculate 2D reconstructed approximation images at first scale $S_{1}(m, n)$ and second scales $S_{2}(m, n)$, i.e. for transform depths of 1 and 2, respectively.

2. Compute the correlation $R_{1,2}(\mathrm{~m}, \mathrm{n})$ for the two scales:

$$
R_{1,2}(m, n)=S_{1}(m, n) \times S_{2}(m, n) .
$$

3. Compute the energy of $R_{1,2}(m, n)$ and $S_{1}(m, n)$ :

$$
\begin{aligned}
E_{S 1} & =\sum S_{1}^{2}(m, n) \\
E_{R 1,2} & =\sum R_{1,2}^{2}(m, n) .
\end{aligned}
$$

In order to make it comparable between the wavelet coefficients and the correlation coefficients, it is necessary to normalise the coefficients:

$$
R_{1,2}^{*}(m, n)=R_{1,2}(m, n) \sqrt{E_{S 1} / E_{R 1,2}} .
$$

4. The wavelet energy $\left|R_{1,2}^{*}(m, n)\right|$ and $\lambda\left|S_{1}(m, n)\right|$ is compared. Here, $\lambda=1$. If $\left|R_{1,2}^{*}(m, n)\right|>\lambda\left|S_{1}(m, n)\right|,(\lambda$ 


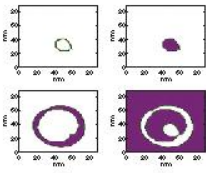

(a)

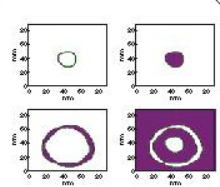

(d)

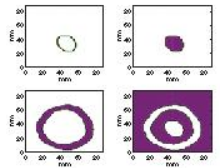

(b)

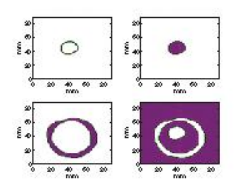

(e)

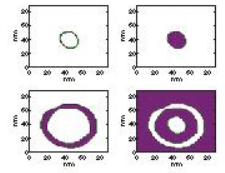

(c)
Fig. 10. Segmented image sets at heights of (a) $5 \mathrm{~mm}$; (b) $6 \mathrm{~mm}$; (c) $7 \mathrm{~mm}$; (d) $8 \mathrm{~mm}$ and (e) $9 \mathrm{~mm}$ from bottom of vial. The four subfigures, clockwise from top left, illustrate the tube edges, tube segments, the air segments (background) and vial segments, respectively.

is a parameter, chosen to be 1 for this experiment), the pixel at $(m, n)$ is extracted as part of a target segment, otherwise it is regarded as background.

The Canny edge detector, combined with Otsu's threshold method [8], is used to perform the final subtraction of each target function edge in the T-ray CT image. It should be noted that the current algorithm only requires the adjustment of a single parameter $\lambda$ in the processing.

\section{EXPERIMENT RESULT}

The algorithm described in Subsection 4.2 is applied to extract segments corresponding to the vial and tube. The extracted approximate sub-images, after computation of the $2 \mathrm{D}$ DWT of the $89 \times 89$ fused images, are shown in Fig. 9(a) and (b); the Daubechies 4 wavelet is used in this case, as mentioned above. The result of the correlation is shown in Fig. 7(a). Figures 10(a)-(e) illustrate the resultant segment subimages corresponding to heights of $5 \mathrm{~mm}$ to $9 \mathrm{~mm}$ from bottom to top. The upper left portion in each subfigure is the edge subimage of the tube; the upper right portion is the segment subimage of the tube; the lower left portion is the vial segment region; the lower right portion is the air segment.

The segmentation quality cannot be directly verified since the explicit ground truth is not known. This situation is typical of tomographic applications, where it is not always possible to cut up the subjects to provide knowledge of the ground truth. Alternatively, a comparison with manual segmentation is undesirable, since that depends on the selected threshold, which cannot afford a standard resultant segment for comparison.

In this experiment, we exploit the fact that the internal structure, the tube, is straight. This implies that the segmented plastic tube positions (see top right of each sub-figure in Fig. 10) should be proportionally displaced from each other, since they correspond to constant increments of $1 \mathrm{~mm}$ in height. To obtain this measurement, we find the centroid of the extracted vial tube for each height (labelled L1-L5) and the resultant $x$ and $y$ locations are plotted in Fig. 11. The achieved linear regression line, with slope of -0.7 and an offset of 81.54 , is used to fit the five measured centroids. The mean

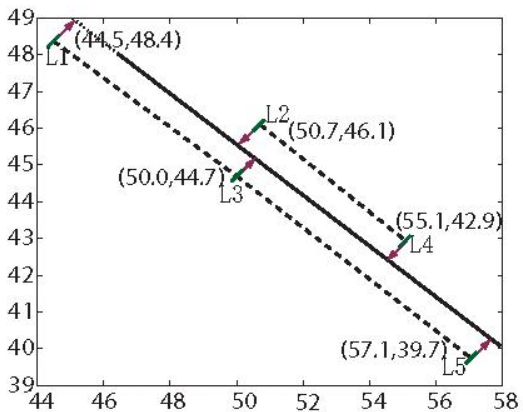

Fig. 11. Illustration of the centroid locations of the target tube segment corresponding to five target heights, L1 to L5. The three dotted lines are the connections of each of the two measured centroids from five target heights; the solid line is to fit the five measured centroids. The $\mathrm{X}$ and $\mathrm{Y}$ axes represent the position of each pixel with the unites of $\mathrm{X}$ and $\mathrm{Y}$ in 'pixel'.

square error of each point to the given solid line is 1.034 . This indicates that our algorithm is capable of locating the centroid of the plastic tube to within a few pixels. Given the number of noise sources and the quality of the reconstructed images, this error is considered to be reasonable.

\section{CONCLUSION}

This paper investigates terahertz computed tomography and the relative application of wavelet based segmentation by fusion. It is a first exploration of automatic T-ray CT target identification within 3D heterogeneous structures. The algorithm successfully segments different target regions and is able to correctly locate the regions to within a few pixels. This provides motivation for future application of classification algorithms for material identification of 3D heterogeneous layers. For future work, it is suggested that the algorithm be characterised further by studying the effect of the segmentation parameter $\lambda$ on the results. In addition, more experimental work is needed to verify the general performance of this algorithm.

\section{REFERENCES}

[1] B. Ferguson, S. Wang, D. Gray, D. Abbott, and X.C. Zhang, "Toward functional 3D T-ray imaging," Physics in Medicine \& Biology, vol. 47, pp. 3735-3742, 2002.

[2] B. Ferguson and D. Abbott, "De-noising techniques for terahertz responses of biological samples," Microelectron. J., vol. 32, no. 12, pp. 943-953, 2001.

[3] J.W. Handley, A.J. Fitzgerald, E. Berry, and R.D. Boyle, "Approaches to segmentation in medical terahertz pulsed imaging," in Proceedings of Medical Image Understanding and Analysis, 2002, pp. 157-160.

[4] C.K. Avinash and S. Malcolm, Principles of Computerized Tomographic Imaging, IEEE Press, New York, U.S.A., 1988.

[5] L. G. Brown, "A survey of image registration techniques," ComputingSurveys, vol. 24, no. 4, pp. 325-376, 1992.

[6] Xiaoxia Yin, Brian W.-H. Ng, Brad Ferguson, Samuel Peter Mickan, and Derek Abbott, "2d wavelet segmentation in 3d t-ray tomography," IEEE Sensors Journal, 2006, Accepted.

[7] S. Mallat, A Wavelet Tour of Signal Processing, Academic Press, San Diego, CA., 1999.

[8] R. C. Gonzalez and R. E. Woods, Digital Image Processing, PrenticeHall, Inc., New Jersey, 2002. 